\title{
Impact of the transport supercurrent on the Josephson effect
}

\author{
S.N. Shevchenko \\ B. Verkin Institute for Low Temperature Physics and Engineering, 47 Lenin \\ Ave., 61103, Kharkov, Ukraine. \\ E-mail: sshevchenko@ilt.kharkov.ua
}

We study the weak link between current-carrying superconductors, both conventional and $d$-wave. The state of the system is controlled by two parameters: the order parameter phase difference $\phi$ and the superfluid velocity $v_{s}$, which parameterizes the parallel to the boundary transport supercurrent which is injected externally. The low-temperature current-phase relations are derived. We consider two models of weak links: a constriction between two conventional superconductors and a plane boundary between two differently orientated $d$-wave superconductors. We show that for some relation between $\phi$ and $v_{s}$ quasiparticles create the current along the boundary which flows in the direction opposite to the transport supercurrent.

\section{INTRODUCTION}

It was demonstrated both experimentally [1]- [3] and theoretically [4], [5] that in some high- $T_{c}$ superconductors the paramagnetic Meissner effect takes place. Namely, at the boundary of a $d$-wave superconductor placed in external magnetic field the current flows in the direction opposite to the diamagnetic Meissner supercurrent which screens the external magnetic field. This countercurrent is carried by the surface-induced quasiparticle states. These nonthermal quasiparticles appear because of the sign change of the order parameter along the reflected quasiparticle trajectory. Such a depairing mechanism is absent in the homogeneous situation. At $T=0$ in a homogeneous conventional superconductor the quasiparticles appear only when the Landau criterion is violated, at $v_{s}>\Delta_{0} / p_{F}$. (Here $v_{s}$ is the superfluid velocity which parameterizes the current-carrying state, $\Delta_{0}$ stands for the bulk order parameter, and $p_{F}$ is the Fermi momentum.)

In a weak link of two superconductors with order parameter phase difference $\phi$, the order parameter can be tuned by $\phi$. In the constriction between two conventional superconductors the proximity order parameter at the contact is given by [6] $\Delta_{\phi}=\Delta_{0}|\cos (\phi / 2)|$. Suppose there is supercurrent tangential to the boundary between two clean conventional superconductors. Namely, let us consider a weak link between current-carrying superconductors. For some values of phase difference $\phi$ and superfluid velocity $v_{s}$, when the condition $v_{s} p_{F}>\Delta_{\phi}$ is satisfied, the quasiparticles appear in the vicinity of the weak link. These quasiparticles create current in the direction opposite to the transport supercurrent in the banks. The aim of the present paper is to study this countercurrent theoretically.

First we consider a weak link between two conventional superconductors as a constriction (see also [7]), which is the simplest situation to demonstrate how the countercurrent appears and how the interface-induced quasiparticle states influence the current through the contact (Josephson current). We then consider a weak link between two differently orientated $d$-wave superconductors (see also [8]). The influence of the transport supercurrent on the state of such a system is interesting in the light of possible applications [9], [10].

\section{THE MODEL. GENERAL REMARKS}

We consider a perfect contact between two clean singlet superconductors. The external order parameter phase difference $\phi$ is assumed to drop at the contact plane at $x=0$. The homogeneous supercurrent flows in the banks of the contact along the $y$-axis, parallel to the boundary. The sample is assumed to be smaller than the London penetration depth so that the externally injected transport supercurrent can indeed be treated as homogeneous far from the weak link. The size of the weak link is assumed to be smaller than the coherence length. Such a system can be quantitatively described by the Eilenberger equation. Taking transport supercurrent into account leads to the Doppler shift of the energy variable by $\mathbf{p}_{F} \mathbf{v}_{s}$. The standard procedure of matching the solutions of the bulk Eilenberger equations at the boundary gives the Matsubara Green's function $\widehat{G}_{\omega}(0)$ at the contact at $x=0[8]$. The component $G_{\omega}^{11}(0) \equiv g_{\omega}(0)$ of $\widehat{G}_{\omega}(0)$ defines current density at the boundary:

$$
\begin{gathered}
\mathbf{j}(0)=4 \pi e N_{0} v_{F} T \sum_{\omega_{n}>0}\left\langle\widehat{\mathbf{v}} \operatorname{Im} g_{\omega}(0)\right\rangle_{\widehat{\mathbf{v}}}, \\
g_{\omega}(0)=\frac{\widetilde{\omega}\left(\Omega_{L}+\Omega_{R}\right)-i \operatorname{sgn}\left(v_{x}\right) \Delta_{L} \Delta_{R} \sin \phi}{\Omega_{L} \Omega_{R}+\widetilde{\omega}^{2}+\Delta_{L} \Delta_{R} \cos \phi},
\end{gathered}
$$

where $N_{0}$ is the density of states at the Fermi level, $\langle\ldots\rangle_{\widehat{v}}$ denotes averaging over the directions of Fermi velocity $\mathbf{v}_{F}, \widehat{\mathbf{v}}=\mathbf{v}_{F} / v_{F}$ is the unit vector in the direction of $\mathbf{v}_{F}, \omega_{n}=\pi T(2 n+1)$ are Matsubara frequencies, $\Delta_{L, R}$ 
stands for the order parameter in the left (right) bank, $\widetilde{\omega}=\omega_{n}+i \mathbf{p}_{F} \mathbf{v}_{s}, \Omega_{L, R}=\sqrt{\widetilde{\omega}^{2}+\Delta_{L, R}^{2}}$.

Analytic continuation of $g_{\omega}(0)$ (i.e. $\omega_{n} \rightarrow-i \varepsilon+0$ ) gives the retarded Green's function; the poles of $g_{\varepsilon}(0)$ determine the energy of the local Andreev states in the system (i.e. bound states at the interface). The directiondependent Doppler shift $\mathbf{p}_{F} \mathbf{v}_{s}$ results in the modification of current-phase dependencies and, particularly, in the appearance of the countercurrent along the boundary. The following sections discuss this phenomenon for the contacts of two conventional and of two $d$-wave superconductors.

\section{WEAK LINK BETWEEN CURRENT-CARRYING CONVENTIONAL SUPERCONDUCTORS}

In the case of a weak link in the form of a constriction between two conventional superconductors with $\Delta_{L}=$ $\Delta_{R}=\Delta_{0}\left(\Delta_{0}=\Delta_{0}\left(T, \mathbf{v}_{s}\right)\right)$ [7] Eqs. 1-2 can be rewritten to study the two components of the current, $j_{x}$ (through the contact), and $j_{y}$ (along the contact):

$$
g_{\omega}(0)=\frac{\widetilde{\omega} \Omega-i \frac{1}{2} \operatorname{sgn}\left(v_{x}\right) \Delta_{0}^{2} \sin \phi}{\widetilde{\omega}^{2}+\Delta_{0}^{2} \cos ^{2} \frac{\phi}{2}} \equiv g_{y}+g_{x},
$$

where we have split $g_{\omega}(0)$ in accordance with the two terms in the numerator, which define $x$ - and $y$ components of the current:

$$
j_{i}(0)=16 \pi e N_{0} v_{F} T \operatorname{Im} \sum_{\omega_{n}>0}\left\langle\widehat{v}_{i} g_{i}\right\rangle_{v_{x}>0, v_{y}>0} .
$$

Here $\Omega=\sqrt{\widetilde{\omega}^{2}+\Delta_{0}^{2}}, i=x, y$. We denote $j_{x}(0) \equiv j_{J}$ and detach transport supercurrent density $j_{T}$ in $j_{y}(0)$, introducing $\widetilde{j}=j_{y}(0)-j_{T}$.

At $T=0$, from Eq.4 after integration we obtain (see also Fig.1) at $p_{F} v_{s}<\Delta_{\phi}: j_{J}=j_{J}^{(0)}=$ $j_{c, 0} \operatorname{sgn}\left(\cos \frac{\phi}{2}\right) \sin \frac{\phi}{2}$ and $\widetilde{j}=0$; at $\Delta_{\phi}<p_{F} v_{s}<\Delta_{0}$ :

$$
\begin{gathered}
j_{J}=j_{J}^{(0)}\left(1-\frac{2}{\pi}\left(\arccos \beta-\beta \sqrt{1-\beta^{2}}\right)\right), \\
\widetilde{j}=j_{c, 0} \sin \frac{\phi}{2}\left(1-\beta^{2}\right), \beta=\frac{\Delta_{\phi}}{p_{F} v_{s}}, \Delta_{\phi}=\Delta_{0}\left|\cos \frac{\phi}{2}\right|,
\end{gathered}
$$

where $j_{c, 0}=\frac{\pi}{2}|e| N_{0} v_{F} \Delta_{0}\left(T=0, v_{s}=0\right)$ is the critical Josephson current at $T=0$ and $v_{s}=0$; for $p_{F} v_{s}<\Delta_{0}$ the transport supercurrent is linear in $v_{s}$ : $j_{T}=\frac{2}{3} e N_{0} v_{F} p_{F} v_{s}$. The condition $p_{F} v_{s}>\Delta_{\phi}$ means that $\phi \in\left(\phi_{1}, \phi_{2}\right)$, where $\phi_{1}=2 \arccos \frac{p_{F} v_{s}}{\Delta_{0}}, \phi_{2}=2 \pi-\phi_{1}$.

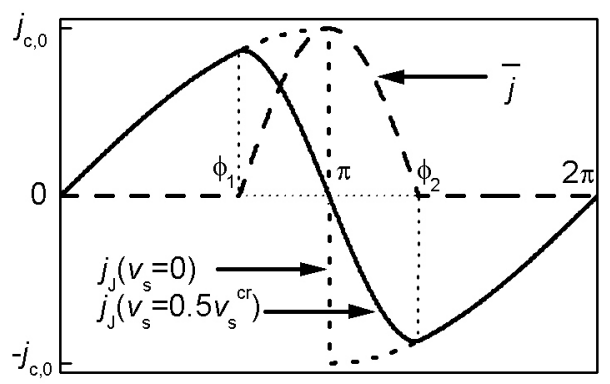

FIG. 1. Zero-temperature current-phase dependence for Josephson current $j_{J}$ (both in the absence and in the presence of the transport supercurrent) and the countercurrent $\widetilde{j}$. The value of the transport supercurrent is chosen to be half of the critical current (at $\left.v_{s}=0.5 \Delta_{0} / p_{F}\right)$.

Thus, at $p_{F} v_{s}<\Delta_{\phi}$ the Josephson current is the same as in the absence of the transport supercurrent and the current density along the contact is equal to the transport supercurrent density. At $p_{F} v_{s}>\Delta_{\phi}$ for a fixed value of $\phi$, the Josephson current $j_{J}$ is suppressed by the transport supercurrent (compared to the Josephson current in the absence of the transport supercurrent): $j_{J}\left(v_{s}\right)<j_{J}\left(v_{s}=0\right)$, and the countercurrent $\widetilde{j}$ appears so that the total tangential current density at the contact $j_{y}(0)$ consists of the transport supercurrent $j_{T}$, carried by the condensate, and of the countercurrent $\widetilde{j}$, carried by nonthermal interface-induced quasiparticles. If there is a current at the contact which flows in the direction opposite to the direction of the current far from the contact (when $\tilde{j}>j_{T}$ ), then the current distribution pattern contains vortex-like formations (see also [7]).

The dependence of the countercurrent $\left.\widetilde{j}\left(v_{s}\right)\right|_{\phi=\pi}$ on $v_{s}$ at $T \neq 0$ can be deduced from the fact that the current increases linearly with $v_{s}$ for $v_{s}<T / p_{F}$ and goes to zero when $v_{s}$ tends to the critical value $v_{s}^{c r}(T)\left(v_{s}^{c r}(0) \simeq\right.$ $\left.p_{F} / \Delta_{0}\right)$.

Our simple model allows straightforward generalizations. For example, consider the situation when the boundary transparency is not one, $D \neq 1$ [11]. Then the proximity gap is $\Delta_{\phi}=\Delta_{0} \sqrt{1-D \sin ^{2}(\phi / 2)}$ and the condition of the appearance of the countercurrent (which is $p_{F} v_{s}>\Delta_{\phi}$ at $\left.T=0\right)$ at $D \neq 1$ is the stronger condition than the condition at $D=1$. At $T=0$ and with given $v_{s}$ and $\phi$ the countercurrent appears for transparent enough junctions with $D>D_{c}\left(v_{s}, \phi, T=0\right)=$ $\left[1-\left(\frac{p_{F} v_{s}}{\Delta_{0}}\right)^{2}\right] / \sin ^{2} \frac{\phi}{2}$.

Eq.3 determines the energy of Andreev states: $\varepsilon_{A}=$ $\pm \Delta_{\phi}-\mathbf{p}_{F} \mathbf{v}_{s}$. Thus there are transport current induced zero-energy states which are characterized by the values $\phi$ and $v_{s}$. At $T=0$ the conductance measures the excitation spectrum of superconductor at the interface [12], 
[5]; the zero-energy states are known to be responsible for the zero-bias conductance peak (ZBCP) [5], [13]. At $\phi=0, \mathrm{ZBCP}$ is expected only in the narrow interval of the value of the transport supercurrent (when there are zero-energy states): $\Delta_{0}<p_{F} v_{s}<1.03 \Delta_{0}$ [14]. In the system under consideration, where the proximity gap $\Delta_{\phi}$ is smaller than the bulk value $\Delta_{0}$, ZBCP is expected even for smaller values of $v_{s}: \Delta_{\phi}<p_{F} v_{s}<1.03 \Delta_{0}$. The observation of ZBCP can be proposed as a test of interface-induced transport-current-dependent quasiparticles states [5]. Similar conclusions about a conductance peak due to the zero-energy bound states were done in [15] for the ac Josephson effect in the point contact with phase difference $\phi$ close to $\pi$.

The appearance of the countercurrent can be understood as the response of the weak link with negative selfinductance to the externally injected transport supercurrent. The state of the junction in the absence of the transport supercurrent at $T=0$ is unstable at $\phi=\pi$ from the point of view that small deviations $\delta \phi= \pm 0$ change $j_{J}$ from 0 to $\mp j_{c, 0}$. As was shown above, the response of the Josephson junction to small transport supercurrent at $\phi=\pi, T=0$ produces the countercurrent $\widetilde{j}=j_{c, 0}$. It is similar to the equilibrium state with the persistent current in 1D normal metal ring with strong spin-orbit interaction: there is degeneracy at $T=0$ and $\phi=\pi$, and the response of the ring is different at $\delta \phi \neq 0$ or $B \neq 0$, where $B$ is the effective magnetic field which enters in the Hamiltonian through the Zeeman term (which breaks time-reversal symmetry) [16]. The degeneracy is lifted by small effective magnetic field so that the persistent current rapidly changes from 0 to its maximum value. In the case of the weak link between two superconductors in the absence of the transport supercurrent there is degeneracy between $+p_{y}$ and $-p_{y}$ zero-energy states; both the time-reversal symmetry breaking by the surface (interface) order-parameter and the Doppler shift (due to the transport supercurrent or magnetic field) lift the degeneracy and result in the surface (interface) current [2], [3].

\section{WEAK LINK BETWEEN CURRENT-CARRYING $D$-WAVE SUPERCONDUCTORS}

Here we consider the weak link between two $d$-wave superconductors whose axes form an angle $\pi / 4$ [8]. The link of this type was proposed as a solid state qubit [10]. The equilibrium state of the contact is doubly degenerate at $\phi= \pm \pi / 2$; there is the interface spontaneous current $j_{S}$ in the equilibrium state; the spontaneous currents flow in the opposite directions in the two states: $j_{S}(-\pi / 2)=$ $-j_{S}(\pi / 2)$. One of the ways to control the state of such a system is by means of transport supercurrent. When the relative angle between two superconductors is equal to $\pi / 4$, the current at the contact is described by Eqs.1-2 with $\Delta_{L}=\Delta_{0} \cos 2 \vartheta$ and $\Delta_{R}=\Delta_{0} \sin 2 \vartheta$ (here $\vartheta$ is the azimuthal angle of $\mathbf{v}_{F}$ ). The contact can be realized in a simply connected geometry, such as a grain boundary, or in a doubly-connected geometry such as a ring (when the phase difference is controlled by the magnetic flux piercing the ring). Correspondingly we consider here two situations: $\phi=0$ (no external phase difference) and $\phi=$ $\pm \pi / 2$ (in the ground state of the contact). The effect of the transport supercurrent on the current parallel to the boundary at $\phi=0$ is shown at Fig.2a.
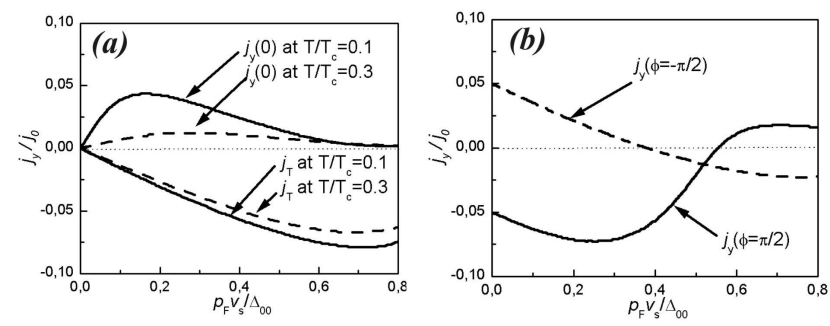

FIG. 2. (a) tangential current density $j_{y}$ in the banks, $j_{T}=j_{y}(\infty)$, and at the contact plane, $j_{y}(0)$, at $T=0.1 T_{c}$ (solid lines) and $T=0.3 T_{c}$ (dashed lines) versus superfluid velocity $v_{s}$; (b) tangential current density at the interface $j_{y}(0)$ for two values of the phase difference versus $v_{s}\left(T=0.1 T_{c}\right)$. Here $\Delta_{00}=\Delta_{0}\left(T=0, v_{s}=0\right), j_{0}=4 \pi|e| N(0) v_{F} T_{c}$.

Making use of Eqs.1-2, we plot the transport supercurrent density $j_{T}$ far from the contact and the tangential component of the current density at the contact $j_{y}(0)$. The latter flows in the opposite direction to the transport supercurrent as in the case of contact of conventional superconductors considered above. For the contact of $d$-wave superconductors, considered in this section, the countercurrent appears for $\phi$ close to 0 and $\pi$ (in contrast to the contact of conventional superconductors, where it appears at $\phi \sim \pi)$. This countercurrent $\widetilde{j}=j_{y}(0)-j_{T}$, which is carried by the quasiparticles, at low temperature rapidly grows at small $v_{s}$ as a function of $v_{s}$ until it becomes equal to the critical value in accordance with what was in detail described in [4]. We also note that at $T=0.3 T_{c}$ our results are similar to the results of [5], presented there in Fig.2(a), where the dependence of the tangential current density on the distance from the boundary for $d$-wave superconductor was plotted. At this point our conclusions for the existence of the countercurrent agree with the previous theoretical results [4], [5] which are consistent with experimental results [1]- [3]. However, to the best of our knowledge, there was no similar study for the case of the weak link (particularly, between conventional superconductors), controlled by both $v_{s}$ and $\phi$, where the countercurrent along the boundary can appear, as described here.

At $\phi= \pm \pi / 2$ (see Fig. 2b) the transport supercurrent removes the degeneracy when $j_{y}(0)= \pm j_{S}$. The 
dependence of $j_{y}(0)$ on $v_{s}$ is nonlinear. The resulting tangential current is not always the sum of the spontaneous current $j_{S}$ and the transport supercurrent $j_{T}$ : $j_{y}(\phi=-\pi / 2) \simeq j_{S}+j_{T} ; j_{y}(\phi=\pi / 2) \simeq-j_{S}+j_{T}$ for $v_{s}<0.2 \Delta / p_{F}$, i.e. until $\left|j_{y}\right|<j_{T}^{\max }$.

\section{DISTRIBUTION OF THE CURRENT IN THE VICINITY OF THE CONTACT}

To illustrate the spatial distribution of the current density in the vicinity of the contact we study the case of ballistic point contact between d-wave superconductors (see Fig. 3, where thick lines denote the impenetrable partition between the superconducting banks). The current distribution pattern in the vicinity of the point contact between conventional superconductors was considered in $[7]$.
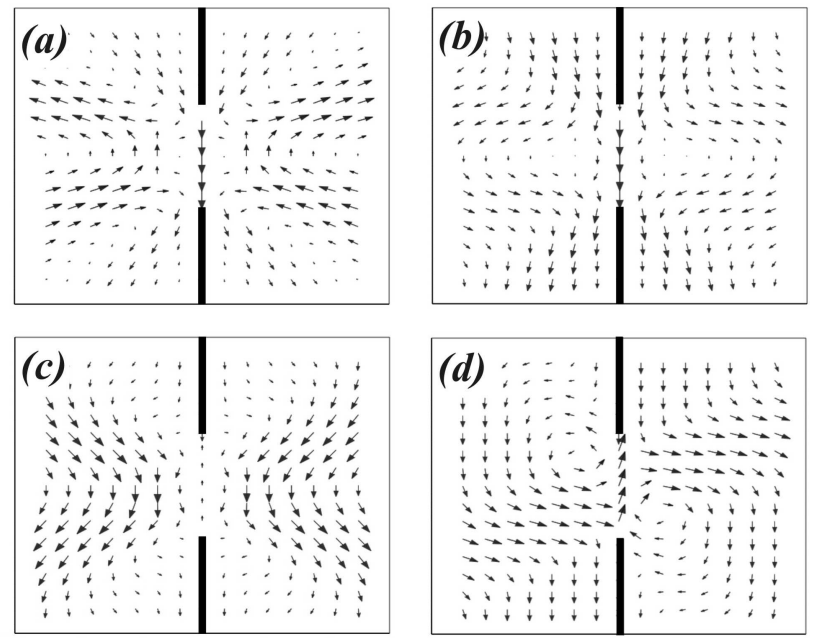

FIG. 3. Distribution of the current density in the vicinity of the contact for (a) $q=0, \phi=\pi / 2$; (b) $q=0.2$ and $\phi=\pi / 2$, (c) $q=0.2$ and $\phi=3 \pi / 2$; (d) $q=0.2$ and $\phi=\pi$; here $q=p_{F} v_{s} / \Delta_{0}\left(T=0, v_{s}=0\right)$.

Although the condition that the contact size is smaller than the coherence length $a<\xi_{0}$ is hardly realizable for high- $T_{c}$ superconductors, we consider this model as an illustrative case to show: (a) how the current is distributed in the ground state of the contact; (b)-(c) how the transport supercurrent modifies the current distribution in the ground state (qualitatively, the resulting current is a sum of what was in the absence of $v_{s}$ and of the transport current); (d) how the appearance of the countercurrent results in the vortex-like current distribution.

\section{CONCLUSION}

The weak link between current-carrying conventional and $d$-wave superconductors has been studied. In such a system it is interesting to study the coexistence of currents of different origin, both transport and interference, or in other words, to understand how current-carrying states of the banks mix non-locally in the vicinity of the contact. The system is also interesting because of possible applications: in the Josephson transistor with controlling parameters $\phi$ and $v_{s}$ governed by external magnetic flux and the transport supercurrent [9], and in solid-state qubits, based on a contact of $d$-wave superconductors [10]. The current at the contact (i.e. its components through the contact and along the contact plane) is controlled by the values of $\phi$ and $v_{s}$. For a particular relation between $\phi$ and $v_{s}$ the countercurrent along the contact appears. This countercurrent is carried by the interface-induced quasiparticles which appear due to the coexistence of the transport supercurrent and the proximity gap. When the countercurrent density is larger than the transport supercurrent density there are vortexlike formations in the current distribution in the vicinity of the contact.

\section{ACKNOWLEDGMENTS}

The author is grateful to Yu.A. Kolesnichenko and A.N. Omelyanchouk for their guidance and help during this research. The author also thanks E.V. Bezuglyi for helpful discussions and P.K. Kovtun for careful reading of the manuscript.

[1] W. Braunisch et al., Phys. Rev. Lett. 68, 1908 (1992).

[2] H. Walter et al., Phys. Rev. Lett. 80, 3598 (1998).

[3] E. Il'ichev et al., Phys. Rev. B 68, 014510 (2003).

[4] S. Higashitani, J. Phys. Soc. Jpn. 66, 2556 (1997).

[5] M. Fogelstrom, D. Rainer, and J.A. Sauls, Phys. Rev. Lett. 79, 281 (1997).

[6] I.O. Kulik and A.N. Omelyanchouk, Fiz. Nizk. Temp. 4, 296 (1978) (Sov. J. Low Temp. Phys. 4, 142 (1978)).

[7] Yu.A. Kolesnichenko, A.N. Omelyanchouk, and S.N. Shevchenko, Phys. Rev. B 67, 172504 (2003).

[8] Yu.A. Kolesnichenko, A.N. Omelyanchouk, and S.N. Shevchenko, Fiz. Nizk. Temp. 30, 288 (2004) (Low Temp. Phys. 30, 213 (2004)).

[9] F.K. Wilhelm, G. Shön, and A.D. Zaikin, Phys. Rev. Lett. 81, 1682 (1998); E.V. Bezuglyi, V.S. Shumeiko, and G. Wendin, preprint cond-mat/0303432.

[10] L.B. Ioffe et al., Nature 398, 679 (1999); A. Blais and A.M. Zagoskin, Phys. Rev. A 61, 042308 (2000); A.M. Zagoskin, J. Phys.: Condens. Matter 9, L419 (1997).

[11] G. Rashedi and Yu.A. Kolesnichenko, Phys. Rev. B 69, 024516 (2004).

[12] C. Duke, "Tunneling in solids", Academic Press, NY (1969). 
[13] C.-R. Hu, Phys. Rev. Lett. 72, 1526 (1994).

[14] D. Zhang, C.S. Ting, and C.-R. Hu, preprint condmat/0312545.

[15] M.H.S. Amin, Phys. Rev. B 68, 054505 (2003).

[16] T.-Z.Qian, Y.-S. Yi, and Z.-B. Su, Phys. Rev. B 55, 4065 (1997); V.A. Cherkassky, S.N. Shevchenko, A.S. Rozhavsky, I.D. Vagner, Sov. J. Low Temp. Phys. 25, 541 (1999); S.N. Shevchenko, Ph.D. thesis, Inst. for Low Temp. Phys. \& Eng., Kharkov (2003). 